\title{
Techno-political strategies and tools to increase interdisciplinary collaboration and community participation around public health policy and the built environment
}

SIGRADI2018 TECHNOPOLITICAS

xxii congresso da sociedade iberoamericana de gráfica digital 22th conference of the iberoamerican society of digital graphics 07|08|09|novembro|2018 iau usp | são carlos | sp br

\author{
Mario Yadir Rendón Sallard \\ Universidad de Sonora | México | yadir42@hotmail.com \\ Elsa Concepción Cornejo Vucovich \\ El Colegio de Sonora | México | ecornejo@colson.edu.mx
}

\begin{abstract}
In order to impact individual behaviors that contribute to risk factors for chronic disease, a multi-disciplinary and inter-institutional working group was established to foster creative ways to increase community participation in public policymaking using the socio-ecological model as a point of reference and community-based participatory research methods to define priority issues and strategies, including techno-political tools such as the creation of a public information repository, community mapping, educational and awareness campaigns, and the use of social media to engage with policymakers.
\end{abstract}

Keywords: Built environment; Health promotion; Chronic disease prevention; Community participation.

\section{INTRODUCCIÓN}

Las enfermedades crónicas no transmisibles como la diabetes y la hipertensión actualmente son la primera causa de muerte en México (Gutiérrez et al. 2012) y en muchas otras partes del mundo. Están asociadas con factores de riesgo como el sedentarismo y el consumo de comida chatarra, comportamientos individuales que a su vez son influidos por el entorno del individuo, incluyendo el ambiente construido. Reconociendo la necesidad de contribuir al estudio de los factores de riesgo modificables de las enfermedades crónicas no transmisibles a nivel mundial, un objetivo principal de la Organización Mundial de la Salud es promover intervenciones dirigidas a disminuir el uso del tabaco, las dietas no saludables y el comportamiento sedentario (OMS 2008), no sólo a nivel individual sino a nivel comunitario y de políticas públicas.

Desde una perspectiva socio-ecológica de la salud —que considera factores que impactan la salud no sólo a nivel individual sino también a nivel interpersonal, organizacional, comunitario y de políticas públicas-, se entiende que el diseño urbano y el ambiente construido tienen el potencial de generar un impacto en el comportamiento individual y en los factores de riesgo para la prevención de las enfermedades crónicas y la promoción de la salud. Este artículo presenta los resultados de la primera etapa de actividades de un grupo de trabajo que reúne a personas de instituciones académicas, agencias gubernamentales, y organizaciones de la sociedad civil. Esta primera etapa incluye el establecimiento de vías de comunicación que permiten una relación colaborativa entre los diferentes actores, así como una mayor participación de los usuarios de los servicios de salud y la ciudadanía en general. También describe el uso de tecnologías innovadoras tanto para la evaluación socio-ecológica de factores de riesgo como para el diseño y construcción de comunidades saludables. Y finalmente identifica las vías posibles para continuar el trabajo iniciado, incluyendo la aplicación de nuevas tecnologías y gestión de recursos.

\section{METODOLOGÍA}

Con el fin de explorar el vínculo entre el ambiente construido y la prevención de las enfermedades crónicas no transmisibles, así como contribuir a mejorar las políticas de diseño urbano y promoción de la salud en una ciudad del norte de México, se estableció un grupo de trabajo que inicialmente estuvo integrado por investigadores de instituciones académicas, y posteriormente involucró a funcionarios de agencias gubernamentales y ciudadanos que integran organizaciones de la sociedad civil, entre otros actores. El grupo de trabajo inició con seminarios exploratorios interdisciplinarios (desde la arquitectura, las ciencias sociales y la salud pública) para establecer temas prioritarios con un enfoque socio-ecológico, para después utilizar métodos de investigación-acción participativa para diseñar actividades y materiales que permitieron la evaluación del diseño de la comunidad estudiada y su impacto en factores de riesgo para la salud, la identificación de mejores prácticas en el diseño urbano y el ambiente construido para promover la salud, y la identificación de estrategias y elaboración de herramientas tecnopolíticas que apoyan el trabajo interdisciplinario y colaborativo que se requiere para tener un impacto en el diseño del ambiente construido de una comunidad, así como para incrementar la participación ciudadana en la elaboración de políticas públicas de diseño urbano y promoción de la salud. 


\section{EL MODELO SOCIO-ECOLÓGICO}

Utilizamos el modelo socio-ecológico como enfoque global de las actividades del grupo de trabajo para dar cuenta que la salud está influenciada por factores que operan a nivel individual, interpersonal, organizacional, comunitario y de sistemas (Stokols 1996). Si bien en México los esfuerzos para prevenir las enfermedades crónicas no transmisibles históricamente se han centrado en programas educativos del sector salud que operan en el nivel individual brindando información acerca de cómo prevenir enfermedades, cada vez hay un mayor reconocimiento de la importancia de las estrategias poblaciones. Un ejemplo son los impuestos a las bebidas azucaradas y la comida chatarra que han contribuido a disminuir su consumo en México (Batis et al. 2017).

Cohen et al. (2000) identifican cuatro categorías de factores estructurales que contribuyen al cambio de comportamiento individual: 1) la disponibilidad que tienen los consumidores a productos que dañan la salud (como las bebidas azucaradas) o que la protegen (como verduras y frutas frescas; 2) las estructuras físicas que fomentan $u$ obstaculizan el comportamiento deseado (como el diseño urbano) o el diseño de los productos físicos (por ejemplo, equipo que ayuda a personas de la tercera edad con poca movilidad a hacer ejercicio); 3) la presencia o ausencia de políticas públicas y estructuras sociales (incluyendo una cultura que asocia la actividad física con el bienestar y que considera que ser físicamente activo es algo positivo); y 4) los medios de comunicación, que tienen el potencial de cambiar actitudes, creencias y cognición a nivel individual, pero también tienen el potencial de modificar normas sociales.

Stokols (1996) también identifica que en cada uno de los niveles identificados por cualquier modelo socio-ecológico hay "intermediarios" que tienen la capacidad de modificar o influir (para bien o para mal) en los factores que finalmente impactan en la toma de decisiones o la capacidad de acción de los individuos. Como grupo de trabajo asumimos el potencial que tenemos como intermediarios y tratamos de identificar estrategias clave para influir en los temas que identificamos como prioritarios para promover la salud a nivel de la comunidad.

Para fines del grupo de trabajo, nos pareció relevante y accesible utilizar el modelo socio-ecológico aplicado a la promoción de la actividad física que desarrolló Active Canada (Figura 1), que reconoce que, para que un individuo adopte un estilo de vida que incluya el mínimo recomendado de actividad física, no solamente debe cambiar su actitud y adquirir habilidades y conocimiento al respecto, sino que necesita una red social que apoye (o no obstaculice) su cambio de hábito, espacios que hagan posible y accesible la actividad física, y una cultura generalizada que fomente el ejercicio, incluyendo leyes y políticas que pasan por el diseño urbano y los programas de fomento al deporte, entre otras.

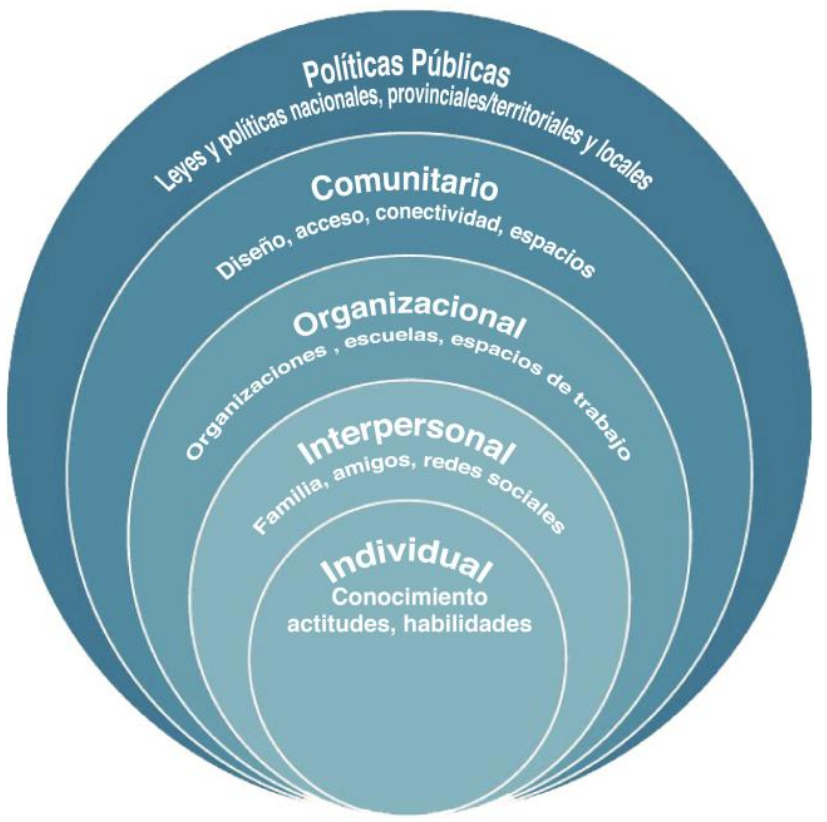

Figura 1: El modelo socio-ecológico aplicado a la promoción de la actividad física (Fuente: Active Canada).

\section{LA INVESTIGACIÓN-ACCIÓN PARTICIPATIVA}

La metodología de la investigación-acción participativa implica la vinculación estratégica entre el equipo de investigación y la comunidad de estudio en un esfuerzo colaborativo, cíclico y reflexivo para identificar temas prioritarios, resolver problemas y mejorar prácticas (Wallerstein y Duran 2010). Los beneficios de este enfoque incluyen la identificación de preguntas de investigación pertinentes y culturalmente apropiadas para la población de estudio, una optimización en la recolección e interpretación de datos, y la facilidad para traducir los hallazgos de investigación en cambios sistemáticos y sustentables (Viswanathan et al. 2004).

La investigación participativa también incluye obligadamente una perspectiva socio-ecológica de la promoción de salud, en la cual se toman en cuenta los comportamientos individuales dentro de un contexto social más amplio, en el que son influidos por escenarios comunitarios, normas socioculturales, contextos institucionales y políticas públicas (Richard, Gauvin y Raine 2011).

Para informar el diseño estratégico de las actividades del grupo de trabajo, se retomó la experiencia aportada por una investigación que actualmente se realiza a nivel estatal, y cuyos objetivos incluyen identificar los factores que facilitan y obstaculizan la adopción de hábitos saludables para la prevención de las enfermedades crónicas y sus complicaciones (Sabo et al. 2018). Gracias a las observaciones de quienes realizan esta investigación, cuyos resultados serán publicados en 2019 , se pudieron identificar factores importantes para la promoción de su salud a nivel individual, familiar, comunitario y social. Estas observaciones fueron una contribución importante que permitió recuperar la voz de la comunidad y sus necesidades sentidas. 


\section{RESULTADOS}

A partir de la primera reunión del grupo de trabajo, en la cual participaron investigadores de las dos instituciones académicas más importantes de la localidad, se hizo una sistematización de experiencias previas para establecer los temas prioritarios de trabajo. De entrada, se decidió que el eje rector de todas las estrategias seria contribuir a crear una visión de una ciudad más humana y más vivible, reconociendo que el diseño urbano y el ambiente construido deben contribuir a satisfacer las necesidades tanto fisiológicas como psicológicas de sus habitantes (Goldhagen 2017).

El grupo de trabajo observó que nos hemos acostumbrado a vivir en una ciudad que carece de planeación estratégica y sin visión de bienestar, y hemos interiorizado que lo que hay es lo que es y que como ciudadanos -aun siendo expertos en el tema 0 funcionarios públicos - tenemos poca capacidad de cambiar una realidad que aparentemente rebasa la capacidad de gestión de un individuo o un grupo de individuos (Jackson 2003). Esto tiene un impacto directo en la motivación que tienen los ciudadanos para hacer cambios de estilo de vida como incrementar la actividad física (Cortés Gil et al. 2016).

Así pues, identificamos que para poder influir en los factores socio-ecológicos que impactan la decisión individual de incrementar la actividad física, y para lograr esa visión de ciudad humana y vivible, tendríamos que trabajar en tres temas urgentes y prioritarios: 1) incrementar el arbolado, así como la creación y el uso de áreas verdes; 2) mejorar el diseño urbano para promover la movilidad y la conectividad, y con ello el acceso a lugares de esparcimiento, así como recuperar zonas históricas y espacios urbanos públicos (sobre todo en el centro histórico de la ciudad que están en desuso o que los programas públicos han abandonado; 3) promover el diseño de fraccionamientos y viviendas dignas y sustentables que fomenten un sentido de comunidad y estén disponibles para todos los sectores sociales y económicos; y 4) contribuir a la promoción de los saberes locales y la creación y difusión del conocimiento en torno a temas de promoción de la salud y ambiente construido.

Una vez establecidos los temas prioritarios, se invitó a otros actores clave a participar en el grupo de trabajo, y se identificaron las herramientas tecnopolíticas más factibles para contribuir a desarrollar cada uno de los temas (Tabla 1).

Entendemos las tecnopolíticas como aquellas herramientas que ha propiciado la era de la información y que promueven la comunicación, la organización y la acción colectivas, siguiendo la definición de Antoni Gutiérrez-Rubí. Consideramos además que estas herramientas tienen el potencial de volver más democrática, menos hegemónica, y más horizontal la generación del conocimiento mismo.
Tabla 1: Temas prioritarios y estrategias tecnopolíticas identificadas por el grupo de trabajo.

\begin{tabular}{|c|c|}
\hline Tema prioritario & Estrategias tecnopolíticas \\
\hline $\begin{array}{l}\text { Incrementar el arbolado } \\
\text { y las áreas verdes } \\
\text { utilizables. }\end{array}$ & $\begin{array}{l}\text { - Mapeo de arbolado y áreas } \\
\text { verdes (con ranking) y } \\
\text { divulgación de resultados por } \\
\text { medios electrónicos. } \\
\text { - Capacitaciones en temas como } \\
\text { permacultura. } \\
\text { - Apoyo técnico para huertos } \\
\text { comunitarios. }\end{array}$ \\
\hline $\begin{array}{l}\text { Mejorar el diseño y el } \\
\text { uso del espacio urbano } \\
\text { (movilidad, conectividad } \\
\text { y recuperación de zonas } \\
\text { históricas y espacios } \\
\text { públicos). }\end{array}$ & $\begin{array}{l}\text { - Mapeo “emocional" realizado } \\
\text { por la comunidad y divulgación } \\
\text { de resultados por medios } \\
\text { electrónicos. } \\
\text { - Consulta colaborativa y } \\
\text { formación de cuadros activistas } \\
\text { para identificar espacios a } \\
\text { rescatar. } \\
\text { - Campañas educativas y de } \\
\text { sensibilización dirigidas a la } \\
\text { comunidad. } \\
\text { - Uso de redes sociales para } \\
\text { promover el acercamiento con } \\
\text { funcionarias y funcionarios } \\
\text { públicos. }\end{array}$ \\
\hline $\begin{array}{l}\text { Promover el diseño de } \\
\text { fraccionamientos y } \\
\text { viviendas dignas y } \\
\text { sustentables que } \\
\text { fomenten un sentido de } \\
\text { comunidad. }\end{array}$ & $\begin{array}{l}\text { - Certificación de vivienda } \\
\text { sustentable que promueva la } \\
\text { interlocución con empresas } \\
\text { constructoras. } \\
\text { - Uso de redes sociales para } \\
\text { promover el acercamiento con } \\
\text { funcionarias y funcionarios } \\
\text { públicos. }\end{array}$ \\
\hline $\begin{array}{l}\text { Contribuir a la creación } \\
\text { y difusión del } \\
\text { conocimiento local en } \\
\text { torno a la promoción de } \\
\text { la salud y el ambiente } \\
\text { construido. }\end{array}$ & $\begin{array}{l}\text { - Seminario multidisciplinario } \\
\text { video-grabado y promovido por } \\
\text { redes sociales. } \\
\text { - Repositorio público online de } \\
\text { bibliografía y documentos. }\end{array}$ \\
\hline
\end{tabular}

Las actividades descritas a continuación actualmente se están llevando a cabo, y los resultados serán presentados en un foro a realizarse en otoño de 2018. Esta presentación de resultados incluirá una evaluación participativa para determinar las acciones siguientes.

\section{INCREMENTAR LAS ÁREAS VERDES}

Según la experiencia del proyecto de investigación que sirve para informar las reflexiones del grupo de trabajo, una de las grandes barreras para hacer actividad física (como caminar o hacer deporte) en espacios exteriores es el clima de la localidad, que se caracteriza por sus temperaturas extremadamente altas. Faltan espacios arbolados en las colonias, e incluso los parques son más bien planchas de tierra o concreto con uno que otro arbusto. Según los expertos en urbanismo del grupo de trabajo, la ciudad no alcanza el porcentaje mínimo de área verde "obligatoria", y la que hay no necesariamente es utilizable (por ejemplo, los camellones se contabilizan como áreas verdes). Por lo tanto, se propuso hacer una 
evaluación tanto cualitativa como cuantitativa de áreas verdes y arbolado, partiendo de un mapeo previo realizado por Lara y García (Lara-Valencia y GarcíaPérez 2013), y esta vez involucrando a estudiantes universitarios/as y asociaciones de vecinos para promover el empoderamiento, la gestión comunitaria y la apropiación ciudadana de los espacios. Se gestionó un taller para realizar mapeo comunitario de áreas verdes, con la intención de que el uso de una tecnología de mapeo sencilla permita que cualquier ciudadano lego pueda usar esta herramienta tecnopolítica para justificar la gestión de recursos públicos para el diseño urbano. Se acordó iniciar con un fraccionamiento al norte de la ciudad que es de reciente creación, para documentar el ejercicio y establecer cuántos recursos se necesitan para replicar la actividad a mayor escala, y para difundir los resultados mediante las redes sociales, otro recurso tecnopolítico que facilita el empoderamiento ciudadano en temas de gestión. Esta actividad, siguiendo la metodología participativa, también sirvió para identificar otras necesidades expresadas por las comunidades, incluyendo el interés por recibir capacitación en temas como la permacultura y la necesidad de tener apoyo técnico para el establecimiento de huertos comunitarios, entre otras que serán programadas en fechas futuras. Todas estas actividades también serán documentadas y divulgadas por redes sociales.

\section{MEJORAR EL DISEÑO Y EL USO DEL ESPACIO URBANO}

Identificamos una necesidad de mejorar el transporte urbano y la articulación de los distintos sectores de la ciudad, particularmente las zonas urbanas centrales, que han sido abandonadas. Esto requiere una visión de "desarrollo comunitario" en su sentido más amplio, considerando las determinantes sociales de la salud y el bienestar, más allá de la pobreza. Syme y Ritterman (2009) han identificado que, aun en condiciones de pobreza, puede florecer la salud si los ciudadanos participan activamente en la gestión de su entorno y tienen un sentido de control sobre lo que les sucede a ellos y a su comunidad. Nos queda claro que las personas que realizan trabajo académico tenemos un papel importante que jugar en la toma de decisiones en cuanto a la infraestructura urbana a nivel local, pero nos queda menos claro cómo fomentar la participación activa y efectiva de la ciudadanía.

Una propuesta es realizar un "mapeo emocional" a manera de consulta, donde los habitantes identifiquen lugares que tienen un significado especial para ellos, y utilizar este ejercicio para colaborar con la comunidad en la formación de cuadros activistas de gestión e interlocución con la administración pública municipal para el rescate de puntos de referencia que han sido abandonados, sobre todo por medio de redes sociales. Asimismo, siguen haciendo falta programas educativos y campañas atractivas de educación y sensibilización dirigidas a la comunidad que promuevan la adopción de hábitos saludables que tengan sentido para esas comunidades. Además, identificamos la necesidad de más campañas que fomenten el concepto de ciudades saludables en un sentido que va más allá de la prevención de enfermedades (por ejemplo, para promover la cultura vial y el respeto a los ciclistas).

\section{PROMOVER EL DISEÑO DE FRACCIONAMIENTOS Y VIVIENDAS DIGNAS}

La planeación urbana de nuestra ciudad deja mucho que desear, y el consenso entre los integrantes del grupo de trabajo es que el diseño urbano lo han determinado las empresas constructoras, cuyo objetivo principal es el lucro. Se planteó la idea de establecer un comité que otorgue un distintivo o una certificación de vivienda sustentable que promueva la interlocución entre expertas y expertos del sector académico, grupos de ciudadanas y ciudadanos, funcionarias y funcionarios del nivel estatal y municipal, y empresas constructoras para establecer criterios de lo que se consideraría una vivienda sustentable y difundirlos para establecer buenas prácticas. Consideramos que este distintivo, que se implementará en un mediano plazo, también es una estrategia tecnopolítica, y que su difusión tiene el potencial de conectar y empoderar a sectores que previamente no interactuaban, potenciados por las oportunidades que ofrece el uso de las tecnologías de la comunicación.

\section{CONTRIBUIR A LA CREACIÓN Y DIFUSIÓN DEL CONOCIMIENTO LOCAL}

Finalmente, consideramos que una de las aportaciones más importantes de este grupo será contribuir a la creación y difusión del conocimiento local en torno a la promoción de la salud y el ambiente construido. De entrada, se estableció una presencia en redes sociales para empezar a difundir las actividades del grupo de trabajo, incluyendo la transmisión por video de los seminarios y talleres, por medio de la fanpage: https://www.facebook.com/Cispac-739769519710287/.

Para darle formalidad e identidad al grupo de trabajo, y para poder invitar a otros colaboradores a participar en una iniciativa más formal que la del grupo de trabajo original, se diseñó un logo y se estableció el Consorcio Interdisciplinario para la Salud Pública y el Ambiente Construido (Figura 2). El grupo de trabajo original sigue proporcionando el liderazgo, pero el establecimiento del CISPAC permite formalizar las actividades de gestión y divulgación.

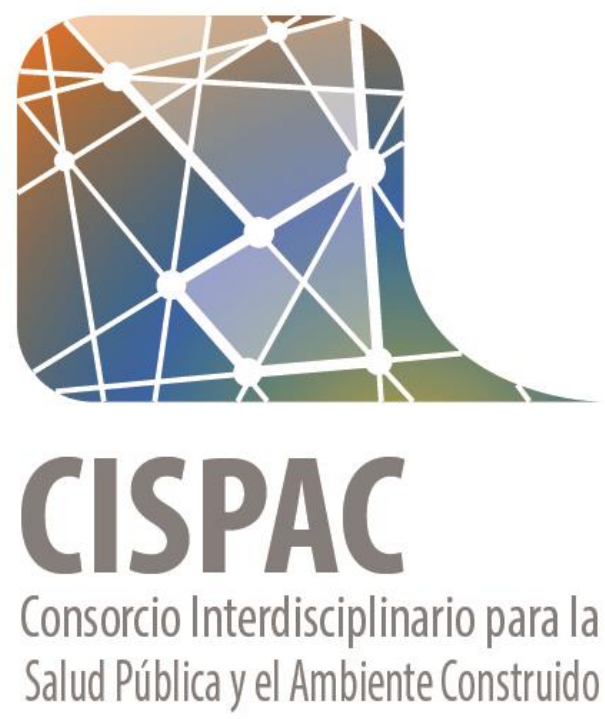

Figura 2: Logo e identidad del grupo de trabajo. 
Además de las actividades de educación, gestión y divulgación descritas, todas las actividades realizadas por el grupo de trabajo y el CISPAC incluyen un componente de investigación-acción participativa, por ejemplo, la documentación de las discusiones en los seminarios y talleres, el uso de encuestas y entrevistas para la evaluación continua, y el involucramiento de los y las participantes en el análisis de los resultados.

Asimismo, la divulgación de las actividades y sus resultados es un componente importante. Para este fin, se estableció un seminario multi-disciplinario permanente para divulgar resultados de investigaciones previas y compartir mejores prácticas, así como identificar oportunidades de colaboración entre las instituciones y agencias participantes. La información recopilada se sistematizará y almacenará en un repositorio de información en línea que incluya bibliografía académica (artículos y tesis), descripción de programas, convocatorias, resultados de investigación, e infografías, entre otros materiales. Este repositorio actualmente está en construcción y la información estará disponible para la consulta pública y gratuita.

\section{CONCLUSIÓN}

Shareck, Frohlich y Poland (2013) han identificado que la creación de ambientes que promueven la salud debe incluir cuatro elementos indispensables: tomar en cuenta los determinantes sociales de la salud, atender las necesidades de los grupos marginados, tratar de efectuar cambios en las cuestiones estructurales del problema de salud a resolver, e involucrar a todos los grupos de interés (stakeholders) en todo momento. Para resolver problemas sociales y de salud pública muy específicos como las enfermedades crónicas no transmisibles como la diabetes y las enfermedades del corazón, no es suficiente diseñar campañas informativas muy puntuales que atiendan los factores de riesgo individuales que las subyacen $\sin$ atender los factores socio-ambientales (Maye 2018). El gran aprendizaje del grupo de trabajo descrito en este artículo fue que se necesita una visión a nivel macro de una ciudad más humana y más vivible que promueve la salud y el bienestar en todos los sentidos. Uno de los grandes retos para este grupo de trabajo será incorporar a más actores clave a sus actividades, al mismo tiempo que se mantenga la metodología participativa y se continúe promoviendo un sentido de pertenencia y control sobre lo que acontece en su comunidad por parte de la ciudadanía, factor indispensable para la promoción de la salud (Syme y Ritterman 2009). Asimismo será necesario identificar indicadores que permitan evaluar el trabajo realizado de manera sistemática para poder informar las políticas públicas con base en evidencia lo más clara posible (Pineo et al. 2017).

\section{AGRADECIMENTOS}

Damos las gracias al equipo de trabajo conformado por la Dra. Brisa Violeta Carrasco Gallegos (Universidad Autónoma del Estado de México), el M.C. Enrique Alejandro Duarte Aguilar y el Dr. Juan Luis Loredo López (Universidad de Sonora), así como a la Dra. Catalina A. Denman (El Colegio de Sonora) y su equipo de investigación.

\section{REFERENCIAS}

Batis, C., Pedraza, L. S., Sánchez-Pimienta, T. G., Aburto, T. C., \& Rivera-Dommarco, J. A. (2017). Energy, added sugar, and saturated fat contributions of taxed beverages and foods in Mexico. Salud Pública de México, 59(5), 512-519. DOI:10.21149/8517.

Cohen, D. A., Scribner, R. A., \& Farley, T. A. (2000). A structural model of health behavior: A pragmatic approach to explain and influence health behaviors at the population level. $\begin{array}{lll}\text { Preventive } \quad \text { Medicine, } & \text { 30(2), }\end{array}$ DOI:10.1006/pmed.1999.0609.

Cortés Gil, J., Orozco Núñez, E., Gatica Domínguez, G., \& Cifuentes, E. (2016). Análisis de algunas barreras del ambiente construido para la actividad física en la Ciudad de Cuernavaca, Morelos, México. Salud, Revista de la Universidad Industrial de Santander, 48(2), 196-205. DOI: dx.doi.org/10.18273/revsal.v48n2-2016004.

Goldhagen, S W. (2017). Welcome to your world: How the built environment shapes our lives. New York: HarperCollins.

Gutiérrez, J. P., Rivera-Dommarco, J., Shamah-Levy, T., Villalpando-Hernández, S., Franco, A., Cuevas-Nasu, L., . . . Hernández-Ávila, M. (2012). Encuesta Nacional de Salud y Nutrición 2012. Resultados Nacionales. Cuernavaca, México: Instituto Nacional de Salud Pública.

Gutiérrez-Rubí, A. (s.f.) Tecnopolítica. El uso y la concepción de las nuevas herramientas tecnológicas para la comunicación, la organización y la acción política colectivas. Disponible en: https://www.gutierrez-rubi.es/wpcontent/uploads/2014/11/Tecnopol\%C3\%ADtica.pdf

Jackson, R. (2003). The Impact of the Built Environment on Health: An Emerging Field. American Journal of Public Health, 93(9), 1382-1384. DOI:dx.doi.org/10.2105/ajph.93.9.1382.

Lara-Valencia, F., \& García-Pérez, H. (2013). Space for equity: socioeconomic variations in the provision of public parks in Hermosillo, Mexico. Local Environment. DOI:10.1080/13549839.2013.857647.

Lowe, M., Whitzman, C., Badland, H., Davern, M., Aye, L., \& Hes, D. et al. (2015). Planning healthy, liveable and sustainable cities: How can indicators inform policy? Urban Policy and Research, 33(2), 131-144. DOI:dx.doi.org/10.1080/08111146.2014.1002606.

Maye, D. (2018). 'Smart food city': Conceptual relations between smart city planning, urban food systems and innovation theory. City, Culture and Society. DOI:dx.doi.org/10.1016/j.ccs.2017.12.001.

National Academies of Sciences, Engineering, and Medicine. (2018). Advancing obesity solutions through investments in the built environment: Proceedings of a Workshop. Washington, DC: The National Academies Press.

Pineo, H., Glonti, K., Rutter, H., Zimmermann, N., Wilkinson, P., \& Davies, M. (2017). Characteristics and use of urban health indicator tools by municipal built environment policy and decision-makers: a systematic review protocol. Systematic Reviews, 6(1). DOI:dx.doi.org/10.1186/s13643-017-0406-x.

Richard, L., Gauvin, L., \& Raine, K. (2011). Ecological Models Revisited: Their Uses and Evolution in Health Promotion Over Two Decades. Annual Review of Public Health, 32, 307326. DOI:10.1146/annurev-publhealth-031210-101141.

Sabo, S., Denman Champion, C., Bell, M. L., Cornejo Vucovich, E., Ingram, M., Valencia, C., . . Rosales, C. B. (2018). Meta Salud Diabetes study protocol: a cluster-randomised trial to reduce cardiovascular risk among a diabetic population of Mexico. BMJ Open, 8(3). DOI:10.1136/bmjopen-2017020762.

Shareck, M., Frohlich, K. L., \& Poland, B. (2013). Reducing social inequities in health through settings-related interventions-a conceptual framework. Global Health Promotion, 20(2), 3952. DOI:10.1177/1757975913486686.

5 
Stokols, D. (1996). Translating Social Ecological Theory into Guidelines for Community Health Promotion. American Journal of Health Promotion, 10(4), 282-298. DOI:10.4278/0890-1171-10.4.282

Syme, L., \& Ritterman, M. L. (2009). The Importance of Community Development for Health and Well-Being. Community Development Investment Review, 5(3), 1-13.
Wallerstein, N., \& Duran, B. (2010). Community-based participatory research contributions to intervention research: the intersection of science and practice to improve health equity. American Journal of Public Health, 100(1), 40-46. DOI:10.2105/AJPH.2009 